\title{
On the white dwarf cooling sequence with extremely large telescopes ${ }^{\star}$
}

\author{
G. Bono ${ }^{1,2}$, M. Salaris ${ }^{3}$, and R. Gilmozzi ${ }^{4}$ \\ ${ }^{1}$ Dipartimento di Fisica, Universita' di Roma Tor Vergata via Della Ricerca Scientifica 1, 00133 Roma, Italy \\ e-mail: bono@roma2 .infn.it \\ 2 INAF, Rome Astronomical Observatory, via Frascati 33, 00040 Monte Porzio Catone, Italy \\ 3 Astrophysics Research Institute, Liverpool John Moores University, 12 Quays House, Birkenhead CH41 1LD, UK \\ ${ }^{4}$ European Southern Observatory, Karl-Schwarzschild-Str. 2, 85748 Garching bei Munchen, Germany
}

Received 16 July 2012 / Accepted 26 October 2012

\section{ABSTRACT}

\begin{abstract}
We present new diagnostics of white dwarf (WD) cooling sequences and luminosity functions (LFs) in the near-infrared (NIR) bands that will exploit the sensitivity and resolution of future extremely large telescopes. The collision-induced absorption (CIA) of molecular hydrogen causes a clearly defined blue turn-off along the WD (WDBTO) cooling sequences and a bright secondary maximum in the WD LFs. These features are independent of age over a broad age range and are minimally affected by metal abundance. This means that the NIR magnitudes of the WDBTO are very promising distance indicators. The interplay between the cooling time of progressively more massive WDs and the onset of CIA causes a red turn-off along the WD (WDRTO) cooling sequences and a well defined faint peak in the WD LFs. These features are very sensitive to the cluster age, and indeed the $K$-band magnitude of the faint peak increases by $0.2-0.25 \mathrm{mag} / \mathrm{Gyr}$ for ages between 10 and $14 \mathrm{Gyr}$. On the other hand, the faint peak in the optical WD LF increases in the same age range by 0.17 ( $V$ band) and 0.15 ( $I$ band) $\mathrm{mag} / \mathrm{Gyr}$. Moreover, we also suggest to use the difference in magnitude between the main sequence turn-off and either the WDBTO (optical) or the WDRTO (NIR). This age diagnostic is also independent of distance and reddening. Once again the sensitivity in the $K$ band $(0.15-0.20 \mathrm{mag} / \mathrm{Gyr})$ is on average a factor of two higher than in the optical bands $(0.10$ [ $V$ band], 0.07 [ $I$ band] mag/Gyr). Finally, we also outline the use of the new diagnostics to constrain the CO phase separation upon crystallization.
\end{abstract}

Key words. stars: evolution - white dwarfs - stars: distances - stars: imaging - globular clusters: general

\section{Introduction}

Among the several relevant contributions by Sandage to stellar astrophysics, the absolute age of Galactic globular clusters (GGCs) has played a major role in astrophysical research. Back in the early fifties he compared the main-sequence turnoff (MSTO) stars in optical color-magnitude diagrams (CMDs) with early cluster isochrones to constrain the age difference both between different clusters (Sandage 1953, 1954) and with the formation and evolution of the Galactic Spheroid (Eggen et al. 1962).

This approach has been and still is the most popular method to constrain the age of Galactic (Buonanno et al. 1998; Stetson et al. 1999; Salaris \& Weiss 2002; Gratton et al. 2003; Zoccali et al. 2003; De Angeli et al. 2005; Di Cecco et al. 2010) and extragalactic (Brown et al. 2004; Milone et al. 2009) stellar systems. Related uncertainties involve the distance modulus (derived either empirically or from theoretical horizontal branch models), the reddening (depending on the methodology followed, Renzini 1991; Bono et al. 2008), the GGC total metallicity (Kraft \& Ivans 2003; Carretta et al. 2009; Asplund et al. 2009), the photometric precision (Stetson 2005), and the efficiency of atomic diffusion in stellar interiors.

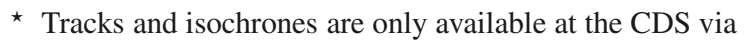
anonymous ftp to cdsarc.u-strasbg. fr $(130.79 .128 .5)$ or via http://cdsarc.u-strasbg.fr/viz-bin/qcat?]/A+A/549/A102
To minimize these uncertainties and employ alternative agedating techniques, three different paths have been followed in the literature.

a) Use of different photometric systems: near-infrared (NIR; Brasseur et al. 2010), Strömgren (Grundahl et al. 1998), Sloan Digital Sky Survey (Di Cecco et al. 2010).

b) Use of different diagnostics for age/distances: luminosity function (LF Zoccali \& Piotto 2001; Richer et al. 2008), the difference in magnitude between the knee displayed by NIR CMDs of low-mass MS stars and the MSTO as an age indicator (Bono et al. 2010).

c) Use of advanced evolutionary phases: asteroseismology of cluster red giant stars (Miglio et al. 2012), and white dwarf (WD) cooling sequences (Richer et al. 2006; De Gennaro et al. 2009; Bedin et al. 2009).

These alternative methods are important since they provide independent constraints on potential systematic errors affecting the "classic" age determinations. Some of these techniques are themselves weakly affected by uncertainties either in distance modulus and reddening, or in the model input physics, or in the assumptions on still uncertain physical mechanisms (e.g., efficiency of atomic diffusion, super-adiabatic envelope convection) that determine the calibration of the MSTO clock.

The identification of new diagnostics becomes even more relevant if they involve advanced evolutionary phases. Absolute 
cluster ages (Richer et al. 2008) and distances (Zoccali et al. 2001) based on WDs, in spite of the intrinsic faintness of these "clocks", have provided this field with a fresh new perspective. Cluster WDs bring forward two decisive positive features: i) their cooling is virtually independent of the progenitor initial metal content (see, e.g. Salaris et al. 2010) and ii) they are the endpoint of the evolution of the large majority of stars, and their properties also provide the opportunity to constrain the modeling of their progenitor evolution (Althaus et al. 2010a, and references therein).

In the following, we discuss new evolutionary features that we identified along the WD isochrones in the NIR bands (Bono 2010, but see also Salaris et al. 2000). These features are located at faint magnitudes $\left(M_{\mathrm{K}} \approx 13-14 \mathrm{mag}\right)$ and their detection in GCs $\left(m_{\mathrm{K}} \approx 26-30 \mathrm{mag}\right)$ is beyond the capabilities of current NIR detectors available at the $10 \mathrm{~m}$ class telescopes. However, this magnitude range will become a unique playground for the next generation of extremely large telescopes (ELTs), namely the European-ELT (E-ELT) ${ }^{1}$, the Thirty Meter Telescope (TMT) ${ }^{2}$, and the Giant Magellan Telescope (GMT) ${ }^{3}$. In Sect. 2 we present the theoretical framework and discuss WD isochrones and LFs both in the optical and in the NIR bands. In the last section we outline the impact that the new generation of ELTs will have on deep and precise NIR photometry of crowded fields.

\section{Theoretical framework}

Figure 1 displays BaSTI isochrones at solar metallicity (scaled solar metal mixture) for 10, 12, and 14 Gyr old populations, including MS, TO, red giant branch and the DA (H-atmosphere) WD cooling sequence (Pietrinferni et al. 2004; Salaris et al. 2010). The left panel shows the isochrones in the ( $V, V-I) \mathrm{CMD}$. We used here updated bolometric corrections for the WD models, employing the recent results by Tremblay et al. (2011) that include the Lyman-alpha profile calculations from Kowalski \& Saumon (2006). The WD isochrones display a clearly defined blue TO (WDBTO, diamonds) whose magnitude and color are correlated with cluster age. The onset of the WDBTO is caused by the pile-up at the bottom of the WD sequence of increasingly more massive - hence with smaller radii - structures. These WDs originate from higher mass progenitors that moved onto the WD sequence during earlier stages of the cluster evolution.

If the distance is independently known (i.e., by means of horizontal branch fitting, MS fitting, RR Lyrae variables), a match of the WDBTO (determined from the magnitude of the LF cutoff in either the $I$ - or the $V$ band; see, e.g., Hansen et al. 2004; Bedin et al. 2009) with models provides the cluster age. If the distance is not known, the difference in magnitude between the MSTO (triangles) and the WDBTO can also be adopted to constrain the absolute age, because the bottom of the WD sequence becomes fainter with age faster than the MSTO. Overall, this latter magnitude difference increases with age by $\sim 0.1 \mathrm{mag} / \mathrm{Gyr}$ in $V$ for ages between 10 and 14 Gyr. In the widely employed $I$-band, the same parameter scales as $\sim 0.07 \mathrm{mag} / \mathrm{Gyr}$. The color difference between MSTO and WDBTO can in principle also be used, but given present observational (the large color spread at the faint limit of the observed WD sequences in GGCs; see, e.g., Bedin et al. 2009) and theoretical uncertainties (colors and $T_{\text {eff }}$ for stars with convective envelopes), it is the magnitude difference that is the better-suited observable.

\footnotetext{
http://www.eso.org/public/teles-instr/e-elt.html

2 http://www.tmt.org/

3 http://www.gmto.org/
}

The key advantages of this "differential" approach are the following:

a) it is independent of uncertainties on the cluster distance and the reddening;

b) it is minimally affected by uncertainties in the photometric zero-point calibrations;

c) the position of the WDBTO is minimally affected by the progenitors' chemical composition;

d) it can be applied over a broad range of cluster ages;

e) it is minimally affected by incompleteness at the faint limiting magnitudes, as long as the WDBTO can be unambiguously identified. The are two main drawbacks:

a) linear and accurate photometry is required over a range of $\approx 12$ mag and

b) the WDBTO is located at very faint magnitudes. This means that even by using ACS/WFPC3 onboard the HST, this approach can only be applied to a few nearby globulars.

The right-hand panel of Fig. 1 displays the same sets of isochrones, but in the NIR $(K, J-K)$ CMD. Here the morphologies of the isochrones are more complicated because of the increasing contribution by collision-induced absorption (CIA) of molecular hydrogen (Hansen 1998; Saumon \& Jacobson 1999) to the opacity in the atmospheres. The strong infrared bands of CIA greatly reduce the infrared flux and increase the flux at shorter wavelengths, producing a typical turn to the blue of NIR colors with decreasing $T_{\text {eff }}$. As a consequence, the lower MS shows a clearly defined knee (position marked with asterisks), that is, at fixed chemical composition, independent of the cluster age. The difference, either in magnitude or in color, of this feature with respect to MSTO stars can also be adopted to constrain the absolute age of star clusters (Bono et al. 2010).

The effect of the CIA opacity on the colors of WD models is similar, but the resulting WD isochrones display an even more complex behavior than MS isochrones. To constrain their properties we followed, a $14 \mathrm{Gyr}$ WD isochrone in the $K, J-K$ CMD at fixed chemical composition together with the underlying WD cooling tracks for stellar masses ranging from $M=0.55$ to $1 M_{\odot}$, see the top panel of Fig. 2. Models plotted in this panel show that WD cooling tracks of increasing mass turn to the blue at almost the same color (corresponding to $T_{\text {eff }} \approx 5000 \mathrm{~K}$ ) and increasingly fainter $K$-band magnitudes. The WD isochrone (red line) at first closely follows a single-mass WD cooling track, the value of this mass being fixed by the adopted initial-to-final mass relation (IFMR). The adopted semi-empirical IFMR is taken from Eq. (1) in Salaris et al. (2009) for initial masses above $0.88 M_{\odot}$, while for lower mass values we adopted a constant value of the final WD mass equal to $0.54 M_{\odot}$ (consistent with the empirical mass determination of bright WDs in the GC M4 by Kalirai et al. 2009). The blue TO that we see in the NIR WD isochrones is in this case caused by the onset of CIA along this single mass cooling track. At fainter magnitudes the WD isochrone becomes populated by progressively more massive WDs. However, these stellar structures are increasingly less blue because of the different cooling speed and onset time of the CIA. This means that after the blue TO, an old NIR WD isochrone also displays a somewhat red TO (WDRTO) due to the appearance of the more massive objects (Bono 2010, but see also Salaris et al. 2000).

It is easy to understand why the brighter part of WD isochrones - in any photometric system - is always populated by essentially single-mass WDs by recalling that at each brightness along the cooling sequence the constraint $t_{\text {iso }}=t_{\text {cool }}+t_{\text {prog }}-$ where $t_{\text {iso }}$ is the age of the isochrone, $t_{\text {cool }}$ the cooling time of 
G. Bono et al.: On the white dwarf cooling sequence with extremely large telescopes

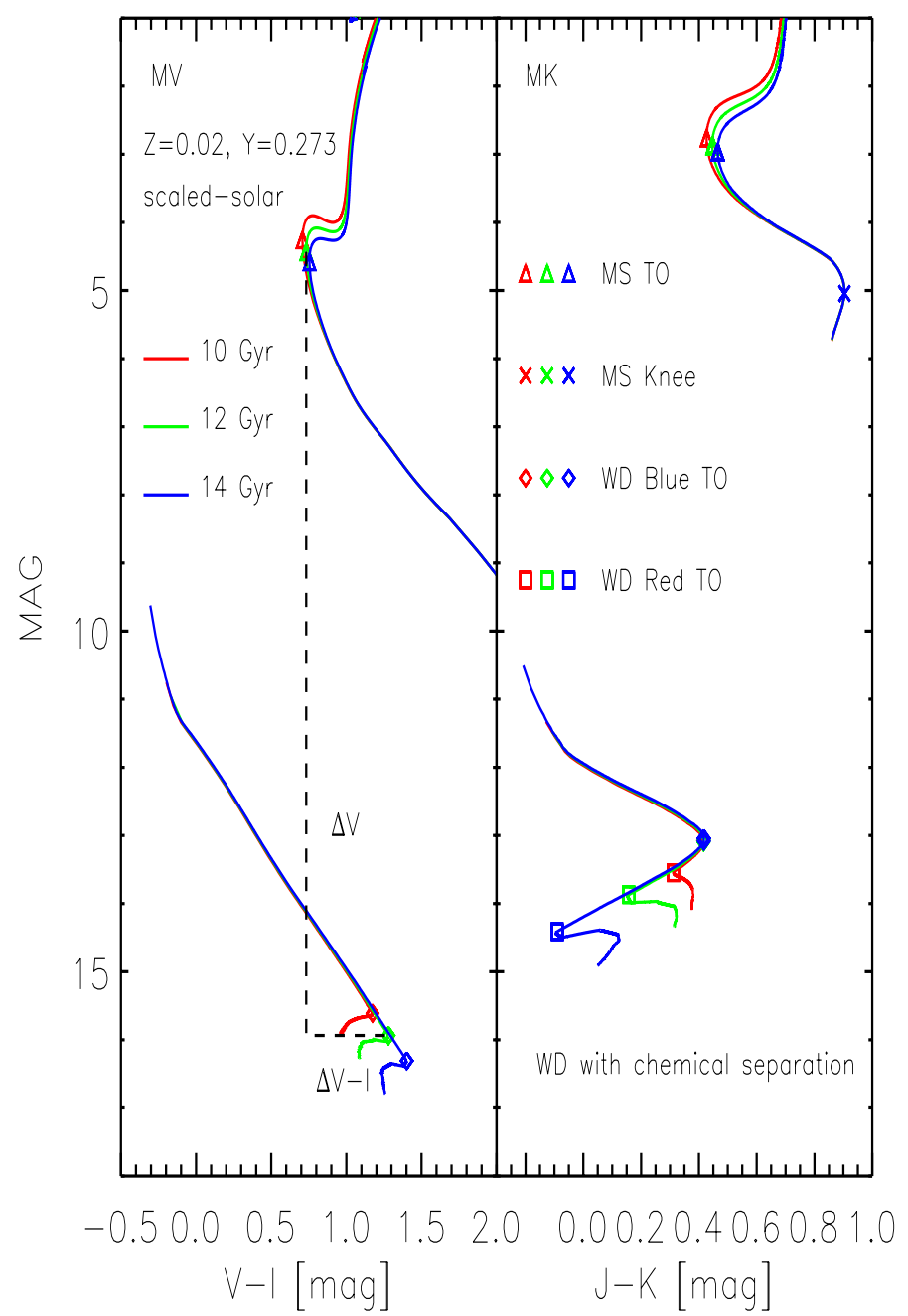

Fig. 1. Left: predicted $(V, V-I) \mathrm{CMD}$ for cluster isochrones at solar chemical composition (see labeled values) and scaled solar chemical mixture. The red, green, and blue solid lines in the top right side of the panel show isochrones of 10,12, and $14 \mathrm{Gyr}$ (BaSTI data base). The triangles mark the MSTO. The red, green, and blue solid lines in the bottom left side of the panel show WD isochrones of 10, 12, and 14 Gyr. The WD isochrones account for chemical separation (Salaris et al. 2010). The diamonds mark the WDBTO. The vertical and horizontal black dashed lines display the difference in magnitude and in color between the 12 Gyr MSTO and the WDBTO. Right: same as left, but in the NIR $(K, J-K)$ CMD. The triangles mark the MSTO, the crosses mark the MS knee (Bono et al. 2010), the diamonds mark the WDBTO, while squares mark the WDRTO.

the "local" WD mass, and $t_{\text {prog }}$ its progenitor lifetime - has to be satisfied. The WD cooling times $-t_{\text {cool }}-$ are very short at the bright end of the sequence, irrespective of the WD mass, and are negligible compared to the cluster age $\left(t_{\mathrm{GC}}\right)$. Therefore, the progenitor age $-t_{\text {prog }}$ - and in turn the progenitor mass and the resulting WD mass, has to be to a very good approximation, constant and very close to the mass at the MSTO. In contrast, the WD cooling time $-t_{\text {cool }}$ - toward the faint end of the cooling sequence becomes a sizable fraction of $t_{\mathrm{GC}}$ and the contribution of the WDs coming from higher mass progenitors (with smaller $t_{\text {prog }}$ ) becomes apparent in the CMD. The exact shape of NIR isochrones is therefore determined by the interplay between cooling times and onset of the CIA.

The bottom panel of Fig. 2 displays NIR WD isochrones for a broad age range. Notice how for ages below $\sim 9$ Gyr the

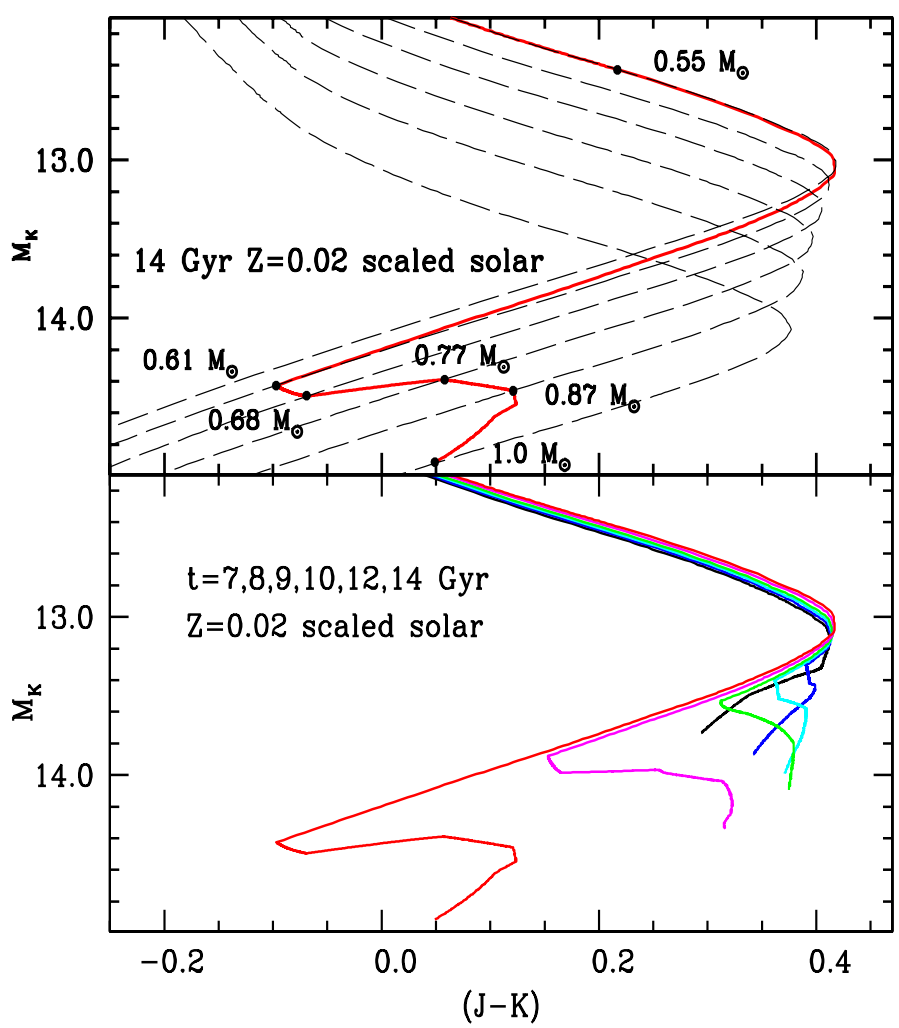

Fig. 2. Top: near-infrared $\left(M_{K}, J-K\right)$ - color-magnitude diagram for a set of white dwarf cooling tracks (long dashed lines) at solar chemical composition $(Z=0.02)$ and scaled solar chemical mixture. The cooling tracks range from $M=0.55$ to $1.0 M_{\odot}$. The red solid line shows the WD isochrone for $14 \mathrm{Gyr}$, while the black filled circles mark different mass values along the isochrone. Bottom: same as top, but for a set of white dwarf isochrones. Lines of different colors display isochrones ranging from 7 (black) to 14 (red) Gyr.

WDRTO disappears. For these ages the blue TO is caused now by the presence of more massive WDs at the bottom of the cooling sequence, as in optical filters. In passing we note that also in optical $V, V-I$ CMDs one would eventually see the effect of CIA on the isochrone colors. However, this happens at ages older than a Hubble time for the BaSTI WD models.

Figure 3 shows the luminosity functions (LFs) in the $I$ - (top) and $K$ band (bottom) for the same age range as displayed in Fig. 2, obtained using a Salpeter mass function $(\alpha=-2.35)$ for the WD progenitors. The interesting feature is that the I-band LF shows a single peak connected with the blue TO at the bottom end of the isochrones in optical filters (see left panel of Fig. 1). On the other hand, the $K$-band LFs display a bright secondary maximum and a faint peak for GC ages older than 7 Gyr. The secondary maximum $\left(M_{K} \approx 13.2 \mathrm{mag}\right)$ is the signature of the blue TO corresponding to the onset of CIA. The position of this feature is age independent (see also the bottom panel of Fig. 2). The faint peak is connected with the red TO at the bottom of the WD sequence and is caused by the appearance of the more massive objects.

This figure highlights several compelling features.

a) The independence of the blue TO absolute $K$ magnitude hence the location of the bright secondary maximum in the LF - for old ages allows us to use this feature as a reference point. In particular, it can be used to determine the cluster distance, since it is independent of age and minimally affected by metallicity. Operationally, observed and theoretical 

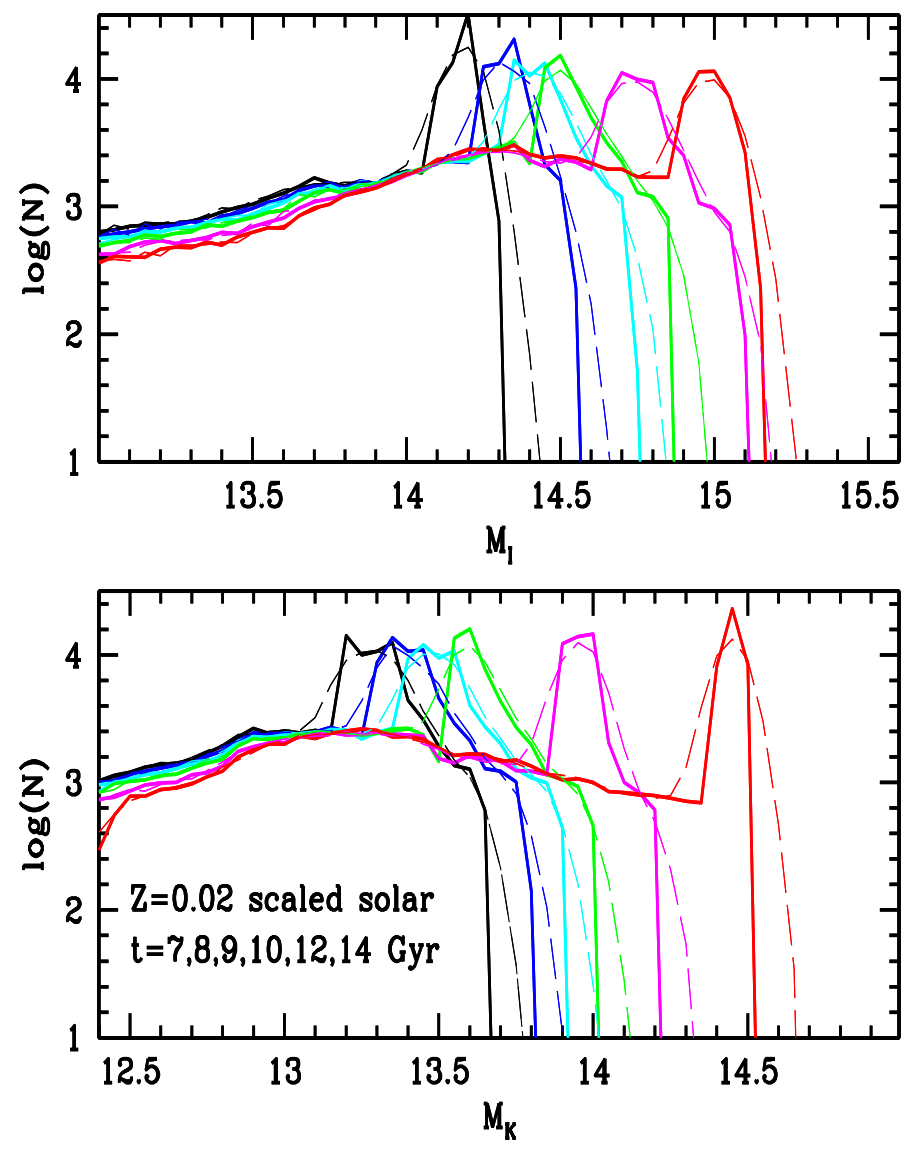

Fig. 3. Top: predicted $I$-band LFs at solar chemical composition and scaled-solar chemical mixture for ages ranging from 7 to $14 \mathrm{Gyr}$ (solid lines). The faint peak at the bottom end of the LF is caused by the WDBTO. Bottom: same as the panel, but for $K$-band LFs (solid lines). The faint peaks are caused by the WDRTO, while the bright secondary maximum centered at $M_{K} \sim 13.2 \mathrm{mag}$ is caused by the CIA-induced blue TO (see text for more details). The dashed lines in both panels display the same LFs, calculated from large synthetic WD samples including a $1 \sigma$ photometric error equal to $0.05 \mathrm{mag}$ in both $I$ and $K$ (see text for details).

LFs can be shifted in magnitude until the bright secondary maximum is matched, thus providing the apparent distance modulus. After this shift in magnitude is applied, the age of the LF can be varied until a match to the faint peak of the LF is achieved, thus estimating the cluster age. For a $1 \sigma$ photometric error of 0.05 mag in $J / K$ bands and cluster ages older than $7 \mathrm{Gyr}$, the fits to the bright secondary maximum of synthetic LFs, including the quoted photometric errors, provide distance moduli with an accuracy better than $0.1 \mathrm{mag}$.

The absolute $K$-band magnitude of the faint peak of the LF (WDRTO) increases by $\sim 0.2 \mathrm{mag} / \mathrm{Gyr}$ for ages between 10 and $12 \mathrm{Gyr}$, and by $\sim 0.25 \mathrm{mag} / \mathrm{Gyr}$ for ages between 12 and $14 \mathrm{Gyr}$. It is worth noting that the absolute $K$ magnitude of the WDRTO - i.e. the bottom end of the LF - is more sensitive to age than the cutoff of the LF in either the $V$ - or $I$ bands. In $V$ the sensitivity is $\sim 0.17 \mathrm{mag} / \mathrm{Gyr}$, whilst in $I$ it is $\sim 0.15 \mathrm{mag} / \mathrm{Gyr}$ in the age range between 10 and $14 \mathrm{Gyr}$. The values of these derivatives are largely independent of the population initial metallicity.

If we use the difference in $K$ magnitude between the MSTO and the cutoff of the WD LF as a distance-independent age indicator, the sensitivity to age is $\sim 0.15-0.20 \mathrm{mag} / \mathrm{Gyr}$, again higher than the same quantity in optical bands.
This enhanced sensitivity to age of the $K$-band LF can greatly improve the internal accuracy of WD-based ages of old star clusters. In Fig. 3 we also show the same $I$ - and $K$-band LFs calculated including a $1 \sigma$ constant photometric error equal to $0.05 \mathrm{mag}$ in both filters. This value is a conservative estimate of the accuracy achievable on GC photometry by the Multi-Conjugate Adaptive Optics assisted instrument MICADO. A MICADO-like camera has been selected as one of the two first light instruments for the E-ELT ${ }^{4}$. Current estimates indicate a point source sensitivity of $5 \sigma$ at limiting magnitudes of $J_{A B} \sim 31$ and $K_{A B} \sim 30$ with five hours of exposure time and with advanced filters ${ }^{5}$. It is interesting to note that similar limiting magnitudes and a similar sensitivity can be reached with NIRCAM@JWST with an exposure time of ten hours ${ }^{6}$, but with a lower spatial resolution.

By adopting the Galactic GC data base by Harris (1996) we found that this new diagnostic could in principle be applied to more than $50 \%$ of GCs if we include all globulars with apparent distance moduli smaller than 15.0-15.5 mag. This sample includes a relevant fraction of bulge and inner halo GCs.

b) The dependence on the initial chemical composition - at fixed IFMR - is extremely weak. The location of the red TO - estimated as the faint peak in the LF - is also weakly affected by the metallicity. The uncertainties affecting the cluster metallicity measurements and the GC metallicity scale are typically smaller than 0.2 dex. It is noteworthy that including the photometric error does not substantially worsen the age-resolving power of the $K$ band LF for ages older than $\sim 10$ Gyr. The initial solar chemical composition adopted to discuss the WD LFs is typical of stellar populations and stellar systems of the Galactic disk. To investigate the WD LFs of typical halo GCs and field stars we also adopted two more metal-poor chemical compositions $([\mathrm{Fe} / \mathrm{H}]=-0.7$ and $[\mathrm{Fe} / \mathrm{H}]]=-2.1)$ and an $\alpha$-enhanced metal mixture $([\alpha / \mathrm{Fe}]=0.4)$. The WD LFs plotted in Fig. 4 were computed by accounting for the individual progenitor lifetimes and demonstrate that the bright secondary maximum located at $M_{K} \sim 13.2$, caused by the CIA opacity, is independent of metallicity. On the other hand, the faint peak in the adopted age range becomes 0.05 mag fainter when moving across the metallicity range of halo GCs $(-0.7 \lessgtr$ $[\mathrm{Fe} / \mathrm{H}]] \lessgtr-2.1)$. The difference in luminosity attains a very similar value when moving from metal-rich $\mathrm{GCs}([\mathrm{Fe} / \mathrm{H}]=$ $-0.7)$ to a solar chemical composition. The decrease in luminosity of the faint peak arises because a decrease in metallicity causes a decrease in the evolutionary lifetime of the WD progenitors. This means that the more metal-poor WD sequences at fixed cluster age and IFMR reach cooler effective temperatures and in turn fainter magnitudes than more metalrich sequences. This evidence further supports the minimal dependence on chemical composition of WD LFs, and indeed a difference of more than two dex in iron abundance causes a difference in the $K$-band LF of at most 0.1 mag. This difference corresponds for old stellar populations to much less than $1 \mathrm{Gyr}$ in terms of age estimates.

\section{We refer to the MICADO web page}

http://www . mpe.mpg.de/ir/micado and to Davies et al. (2010).

5 For a more detailed discussion concerning the band-pass and the OH-suppression filters for MICADO@E-ELT we refer to Guenster et al. (2010).

6 We refer to the NIRCAM web page

http://ircamera.as. arizona.edu/nircam/ and to Rieke (2011). 


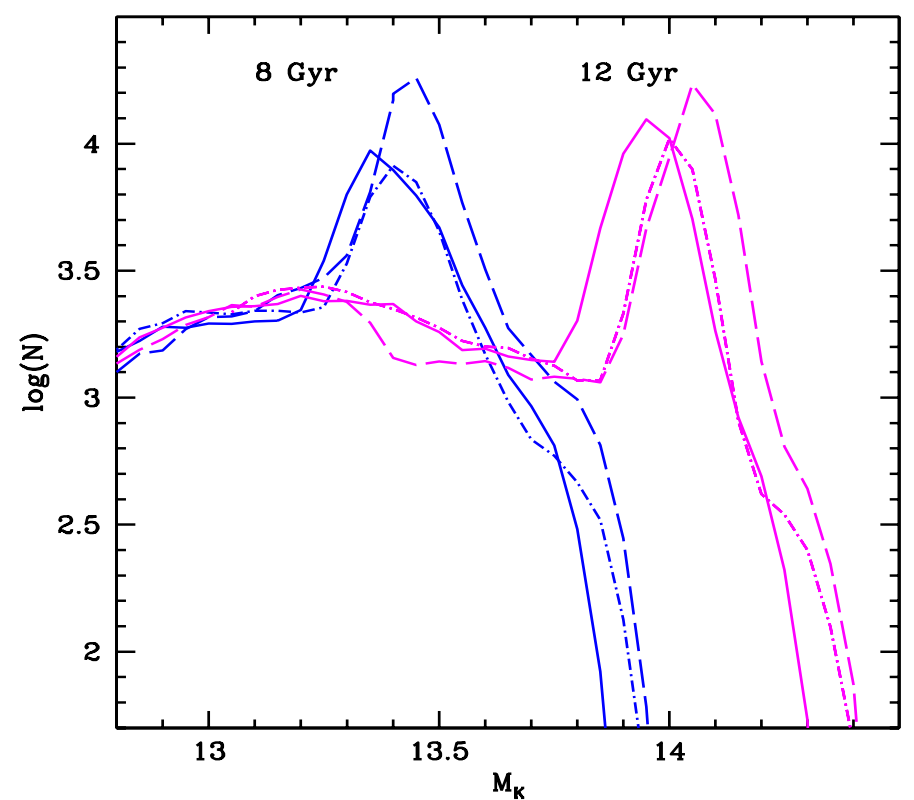

Fig. 4. Same as the lower panel of Fig. 3, but for two ages -8 and $12 \mathrm{Gyr}$ - and three different chemical compositions. The solid lines show the LFs at solar chemical composition, while the dotted-dashed and long-dashed lines display the $\alpha$-enhanced $([\alpha / \mathrm{Fe}]=0.4) \mathrm{LFs}$ for two more metal-poor chemical compositions, $[\mathrm{Fe} / \mathrm{H}]=-0.7$ and $[\mathrm{Fe} / \mathrm{H}]=-2.1$.

c) The position in the NIR CMD of the blue TO depends only on the IFMR (see, e.g. Kalirai et al. 2009; Salaris et al. 2009, and references therein). Changes in the evolving WD mass in this region from $0.55 M_{\odot}$ - the value for the IFMR adopted in BaSTI - to $0.61 M_{\odot}$ (a considerable change, and probably an overestimate of the final mass for typical MSTO masses in GCs) increases the $K$ magnitude of the blue TO by $\sim 0.1 \mathrm{mag}$. If extremely accurate distances are available independently of the cooling sequence, the observed absolute magnitude of this feature may in principle be used to constrain the IFMR for low-mass metal-poor stars.

d) The reason for the enhanced sensitivity to age of the $K$-band WD LF is disclosed by Fig. 5, which shows the run of the bolometric corrections to the $I$ - and the $K$-band $\left(\mathrm{BC}_{I}\right.$ and $\mathrm{BC}_{K}$ ) along the fainter portion of a representative cooling track of a $0.55 M_{\odot}$ model. In general, the $\mathrm{BC}_{K}$ values have a much steeper dependence on $T_{\text {eff }}$ (hence bolometric luminosity) than $\mathrm{BC}_{I}$; the local maximum of $\mathrm{BC}_{K}$ at $T_{\text {eff }} \sim 5000 \mathrm{~K}$ marks the onset of CIA opacity. Beyond this maximum, $\mathrm{BC}_{K}$ drops very fast with decreasing $T_{\mathrm{eff}}$, whilst $\mathrm{BC}_{I}$ continues to steadily increase with decreasing $T_{\text {eff }}$, with a shallower slope. Taking into account this behavior of the bolometric corrections, and given that $M_{X}$ in a generic photometric band $X$ can be written as $M_{x}=M_{\mathrm{bol}}-\mathrm{BC}_{X}$, the dimming rate of $M_{I}$ will necessarily be slower than for $M_{K}$, because of the opposite effect of the corresponding bolometric corrections on the decrease in the bolometric luminosity with time.

Another important consequence of this enhanced agesensitivity is that the effect of CO separation upon crystallization will induce a larger difference - with respect to the case of no separation - in the magnitude of the bottom end of the $K$-band LF (for a given WD isochrone age) compared to the case of filters at optical wavelengths. This is because CO separation causes a slower WD cooling, hence brighter WDs at a fixed (old) population age. Because of the behavior of the bolometric corrections discussed above (see Fig. 5), a

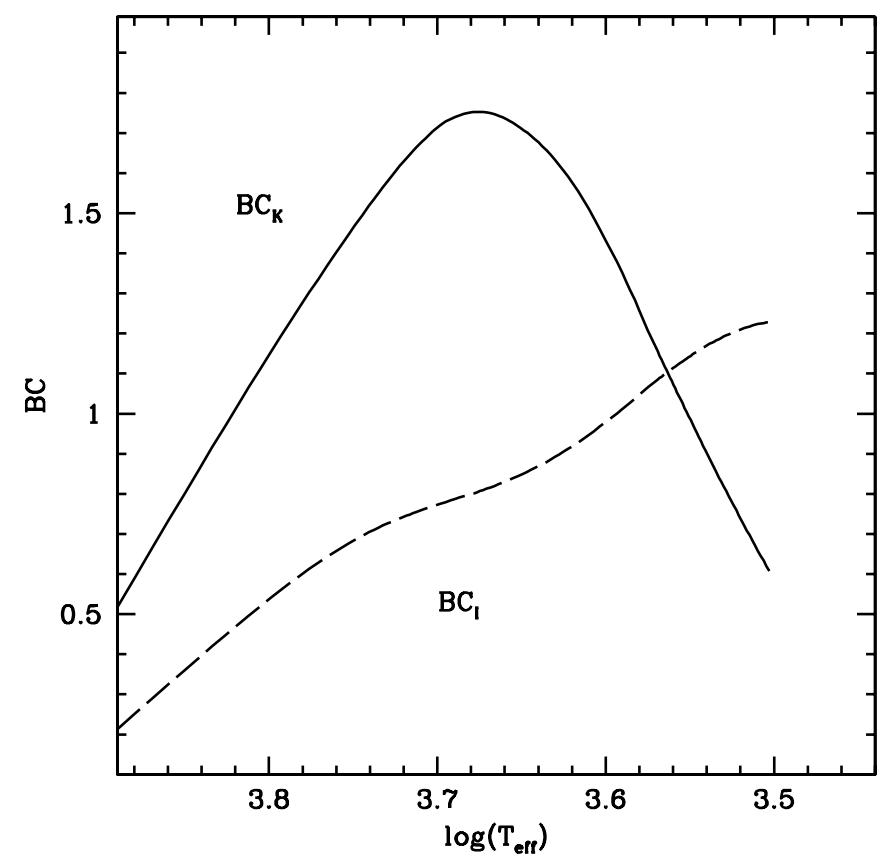

Fig. 5. Bolometric corrections to the $K$ - and $I$ band as a function of $T_{\text {eff }}$ along the $0.55 M_{\odot}$ cooling track. The maximum of the $\mathrm{BC}_{K}$ located at $T_{\text {eff }} \sim 5000 \mathrm{~K}$ marks the onset of CIA opacity.

given difference in bolometric luminosity of the termination of the WD sequences translates into a larger magnitude difference for the $K$-band compared to optical bandpasses.

\section{Conclusions and final remarks}

The use of the next generation of ground-based ELTs and of new space facilities (Gaia, JWST) will bring forward crucial advances in our understanding of the Universe. Whenever either a new instrument, a new wavelength region, or a new observing facility becomes available we are at a classical crossroads, with two alternatives to follow: i) improve the precision of wellknown diagnostics (semantic approach); ii) develop new diagnostics (archetypal approach). The latter route is less trivial, but offers a major reward: the opportunity of identifying new evolutionary features that can provide independent checks on the systematics affecting well-established distance and age indicators.

This latter path is what was followed in the analysis of the inflection point along the low-mass MS in NIR CMDs (Bono et al. 2010). This point is marginally dependent on metallicity and independent of reddening, and can be adopted to provide robust estimates of both cluster ages and distances. The same applies to both blue and red TOs predicted in NIR WD isochrones and LFs, as discussed in this investigation. Once again, they can be adopted to constrain both the cluster ages and distances. Using different diagnostics to constrain the properties of the same stellar system has several decisive advantages:

i) Constraints on systematics - Using additional evolutionary phases provides the opportunity of constraining the input physics and the physical assumptions adopted to construct models for the MS and advanced evolutionary stages. Moreover, diagnostics relying on different evolutionary phases and based on different observables (CMDs, LFs) can be used to constrain the precision of photometric zeropoints and model atmosphere adopted to transform the output of theoretical stellar models into the observational plane. 
The GGC distances can be estimated using several standard candles (MS fitting, tip of the RGB, RR Lyrae, WD cooling sequences, proper motion, eclipsing binaries). Their application to the same system can provide firm constraints on systematics (Bono et al. 2008; Thompson et al. 2010).

ii) Physics laboratories - GGCs are redundant systems (Bono 2010), therefore their intrinsic parameters (age, distance, chemical composition) can be estimated using different observables. The GGC ages can be estimated using different observables (LFs, MSTO, MS knee, WD cooling sequences). Once independent approaches show a global concordance, the advanced evolutionary phases can be adopted to constrain interesting physical phenomena. The opportunity of estimating both the cluster age and the cluster distance using the WD cooling sequences can provide firm constraints on other age indicators. Cluster ages based on the MSTO are still hampered by uncertainties that affect the treatment of gravitational settling of heavy elements (Richard 2002). Unfortunately, recent spectroscopic measurements did not settle this open problem (Thevenin et al. 2001; Gratton et al. 2001; Korn et al. 2006). Accurate and independent age estimates will allow us to perform an empirical calibration of the free parameters currently adopted in dealing with atomic diffusion (Chayer et al. 1995; Wassermann et al. 2010; Althaus et al. 2010a).

This is the first paper of a series. The next step is to constrain the impact that ${ }^{22} \mathrm{Ne}$ sedimentation in high-metallicity progenitors (Althaus et al. 2010b) and $\mathrm{H}_{2}-\mathrm{He}$ CIA (Jørgensen et al. 2000) have on the cooling sequences of WDs with mixed $\mathrm{H} / \mathrm{He}$ atmospheric compositions (Koester 2002; Hansen \& Liebert 2003).

The construction plan of ELTs is already in a mature phase, with the E-ELT having just been approved for construction (McPherson et al. 2012). The other two projects (TMT, GMT) are in a similar construction phase. However, we still need to wait approximately ten years for any of them to achieve first light. While the full application of the diagnostics described in this paper will have probably to wait that long, clearly the current and next generation of instruments in the $10 \mathrm{~m}$ class telescopes will play a crucial role in paving the road for these unique facilities.

Acknowledgements. It is a real pleasure to thank L. Althaus for many useful discussions concerning evolutionary properties of white dwarfs and R. Davies for several thorough discussions concerning MICADO. It is also a pleasure to thank the anonymous referee for his/her pertinent suggestions that helped us to improve the content and the readability of the paper. One of us (G.B.) thanks ESO for support as a science visitor. This work was partially supported by PRININAF 2011 "Tracing the formation and evolution of the Galactic halo with VST" (P.I.: M. Marconi) and by PRIN/MIUR (2010LY5N2T) "Chemical and dynamical evolution of the Milky Way and Local Group galaxies" (P.I.: F. Matteucci).

\section{References}

Althaus, L. G., Corsico, A. H., Isern, J., \& Garcya-Berro, E. 2010a, A\&ARv, 18, 471

Althaus, L. G., Garcya-Berro, E., Renedo, I., et al. 2010b, ApJ, 719, 612
Asplund, M., Grevesse, N., Sauval, A. J., \& Scott, P. 2009, ARA\&A, 47, 481 Bedin, L. R., Salaris, M., Piotto, G., et al. 2009, ApJ, 697, 965

Bono, G. 2010, Mem. Soc. Astron. It., 81, 863

Bono, G., Stetson, P. B., Sanna, N., et al. 2008, ApJ, 686, L87

Bono, G., Stetson, P. B., VandenBerg, D. A., et al. 2010, ApJ, 708, L74

Brasseur, C. M., Stetson, P. B., VandenBerg, Don A., et al. 2010, AJ, 140, 1672

Brown, T., Ferguson, H. C., Smith, E., et al. 2004, ApJ, 613, L125

Buonanno, R., Corsi, C. E., Pulone, L., Fusi Pecci, F., \& Bellazzini, M. 1998, A\&A, 333, 505

Carretta, E., Bragaglia, A., Gratton, R., D’Orazi, V., \& Lucatello, S. 2009, A\&A, 508,695

Chayer, P., Fontaine, G., \& Wesemael, F. 1995, ApJS, 99, 189

Davies, R., Ageorges, N., Barl, L., et al. 2010, in Ground-based and Airborne Instrumentation for Astronomy III, eds. I. S. McLean, S. K. Ramsay, \& H. Takami, SPIE, 7735, 77

De Angeli, F., Piotto, G., Cassisi, S., et al. 2005, AJ, 130, 116

De Gennaro, S., von Hippel, T., Jefferys, W. H., et al. 2009, ApJ, 696, 12

Di Cecco, A., Becucci, R., Bono, G., et al. 2010, PASP, 122, 991

Eggen, O. J., Lynden-Bell, D., \& Sandage, A. R. 1962, ApJ, 136, 748

McPherson, A., Gilmozzi, R., Spyromilio, J., Kissler-Patig, M., \& Ramsay, S. 2012, Messenger, 148, 2

Gratton, R. G., Bonifacio, P., Bragaglia, A., et al. 2001, A\&A, 369, 87

Gratton, R. G., Bragaglia, A., Carretta, E., et al. 2003, A\&A, 408, 529

Grundahl, F., Vandenberg, D. A., \& Andersen, M. I. 1998, ApJ, 500, L179

Guenster, St., Ristau, D., \& Davies, R. 2011, SPIE, 8168, 81681

Hansen, B. M. S. 1998, Nature, 394, 860

Hansen, B. M. S., \& Liebert, J. 2003, ARA\&A, 41, 465

Hansen, B. M. S., Richer, H. B., Fahlman, G. G., et al. 2004, ApJS, 155, 551

Harris, W. E. 1996, AJ, 112, 1487 (2010 edn.)

Jørgensen, U. G., Hammer, D., Borysow, A., \& Falkesgaard, J. 2000, A\&A, 361, 283

Kalirai, J. S., Saul, D. D., Richer, H. B., et al. 2009, ApJ, 705, 408

Koester, D. 2002, A\&ARv, 11, 33

Korn, A. J., Grundahl, F., Richard, O., et al. 2006, Nature, 442, 657

Kowalski, P. M., \& Saumon, D. 2006, ApJ, 651, L137

Kraft, R. P., \& Ivans, I. I. 2003, PASP, 115, 143

Miglio, A., Brogaard, K., Stello, D., et al. 2012, MNRAS, 419, 2077

Milone, A. P., Bedin, L. R., Piotto, G., \& Anderson, J. 2009, A\&A, 497, 755

Pietrinferni, A., Cassisi, S., Salaris, M., \& Castelli, F. 2004, ApJ, 612, 168

Renzini, A. 1991, in Observational Tests of Cosmological Inflation, eds. T. Shanks, A. J. Banday, \& R. S. Ellis (Dordrecht: Klower), 131

Richard, O., Michaud, G., Richer, J., et al. 2002, ApJ, 568, 979

Richer, H. B., Anderson, J., Brewer, J., et al. 2006, Science, 313, 936

Richer, H. B., Dotter, A., Hurley, J., et al. 2008, AJ, 135, 2141

Rieke, M. 2011, in Galaxy evolution: Infrared to millimeter wavelength perspective, eds. W. Wang, J. Lu, Z. Luo, et al. (San Francisco: ASP), 331

Salaris, M., \& Weiss, A. 2002, A\&A, 388, 492

Salaris, M., Garca-Berro, E., Hernanz, M., Isern, J., \& Saumon, D. 2000, ApJ, 544,1036

Salaris, M., Serenelli, A., Weiss, A., \& Miller Bertolami, M. 2009, ApJ, 692, 1013

Salaris, M., Cassisi, S., Pietrinferni, A., Kowalski, P. M., \& Isern, J. 2010, ApJ, 716,1241

Sandage, A. R. 1953, AJ, 58, 61

Sandage, A. R. 1954, AJ, 59, 162

Saumon, D., \& Jacobson, S. B. 1999, ApJ, 511, L107

Stetson, P. B. 2005, PASP, 117, 563

Stetson, P. B., Bolt, M., Harris, W. E., et al. 1999, AJ, 117, 247

Thvenin, F., Charbonnel, C., de Freitas Pacheco, J. A., et al. 2001, A\&A, 373, 905

Thompson, I. B., Kaluzny, J., Rucinski, S. M., et al. 2010, AJ, 139, 329

Tremblay, P.-E., Bergeron, P., \& Gianninas, A. 2011, ApJ, 730, 128

Wassermann, D., Werner, K., Rauch, T., \& Kruk, J. W. 2010, A\&A, 524, A9

Zoccali, M., \& Piotto, G. 2000, A\&A, 358, 943

Zoccali, M., Renzini, A., Ortolani, S., et al. 2001, ApJ, 553, 733

Zoccali, M., Renzini, A., Ortolani, S., et al. 2003, A\&A, 399, 931 